\title{
A Highly Sensitive GFP Activation Assay for Detection of DNA Cleavage in Cells
}

\author{
Ziying $\mathrm{Hu}^{1,2}$, Chengdong Zhang ${ }^{1}$, Daqi Wang ${ }^{1}$, Siqi Gao ${ }^{1}$, Sang-Ging Ong ${ }^{3,4}$, \\ Yongming Wang ${ }^{1,5 *}$ and Wei V. Zheng ${ }^{6 *}$
}

${ }^{1}$ State Key Laboratory of Genetic Engineering, School of Life Sciences, Zhongshan Hospital, Fudan University, Shanghai, China, ${ }^{2}$ Centre for Assisted Reproduction, Shanghai First Maternity and Infant Hospital, Tongji University School of Medicine, Shanghai, China, ${ }^{3}$ Department of Pharmacology, University of Illinois College of Medicine, Chicago, IL, United States, ${ }^{4}$ Division of Cardiology, Department of Medicine, University of Illinois College of Medicine, Chicago, IL, United States, ${ }^{5}$ Shanghai Engineering Research Center of Industrial Microorganisms, Shanghai, China, ${ }^{6}$ Intervention and Cell Therapy Center, Peking University Shenzhen Hospital, Shenzhen, China

\section{OPEN ACCESS}

Edited by:

Puping Liang,

Sun Yat-sen University, China

Reviewed by:

Yujing $L i$,

Indiana University-Purdue University Indianapolis, United States Xin Han,

Nanjing University of Chinese Medicine, China

*Correspondence: Yongming Wang ymw@fudan.edu.cn

Wei $V$. Zheng

zhengw2013@yeah.net

Specialty section:

This article was submitted to Molecular and Cellular Pathology, a section of the journal Frontiers in Cell and Developmental Biology

Received: 06 September 2021

Accepted: 21 October 2021

Published: 11 November 2021

Citation:

Hu Z, Zhang C, Wang D, Gao S,

Ong S-G, Wang $Y$ and Zheng $W V$

(2021) A Highly Sensitive GFP Activation Assay for Detection of DNA

Cleavage in Cells.

Front. Cell Dev. Biol. 9:771248.

doi: $10.3389 /$ fcell.2021.771248
CRISPR/Cas9 nucleases hold great potential for gene therapy, but they frequently induce unwanted off-target cleavage. We previously developed a GFP activation assay for detection of DNA cleavage in cells. Here, we demonstrate two novel applications of this assay. First, we use this assay to confirm off-target cleavage that cannot be detected by targeted deep sequencing in cells before. Second, we use this approach to detect multiple alternative PAMs recognized by SpCas9. These noncanonical PAMs are associated with low cleavage activity, but targets associated with these PAMs must be considered as potential off-target sites. Taken together, the GFP activation assay is a powerful platform for DNA cleavage detection in cells.

Keywords: CRISPR, genome editing, DNA cleavage, off-target, PAM

\section{INTRODUCTION}

The RNA-guided CRISPR/Cas9 system can introduce desired mutations into the genome and therefore has a broad range of research and medical applications (Cong et al., 2013; Hwang et al., 2013; Mali et al., 2013; Xie et al., 2017; Wang et al., 2019a). This system consists of a Cas9 nuclease and a guide RNA (gRNA), which forms a Cas9-gRNA complex, recognizing a target sequence (protospacer) with a downstream protospacer adjacent motif (PAM), and induces a site-specific double-strand break (DSB) (Jinek et al., 2012; Cong et al., 2013; Mali et al., 2013). DSBs are repaired by either non-homologous end joining (NHEJ) or homologous recombination (HR) repair pathway, resulting in desired mutations (Wang et al., 2012; Ran et al., 2013a; Wang et al., 2014). Although the targeting specificity of Cas 9 nucleases is controlled by the 20-nt guide sequence of the gRNA and the presence of a PAM adjacent to the target sequence in the genome, potential off-target cleavage could still occur (Fu et al., 2013; Hsu et al., 2013; Ran et al., 2013b; Fu et al., 2014). Off-target cleavage requires a DNA sequence with certain degrees of homology to the target sequence, followed by a PAM or a noncanonical PAM (Hsu et al., 2013; Lin et al., 2014; Wang et al., 2019a). Off-target mutations can confound interpretation of the experiments and can have implications for the development of therapeutic applications.

The importance of the off-target issue has spurred the development of multiple approaches to identify the frequencies and locations of unintended off-target mutations (Tsai and Joung, 2016). The initial approaches are in silico prediction of potential off-target cleavage sites based on similarity to the intended target site followed by the targeted experimental assessment of indel mutations at those 
A

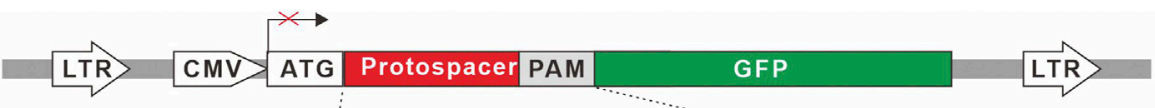

off-target sequence AAGTCCGAGGAGAGGAAGAA $\underline{\underline{A G G}}$

on-target gRNA GAGTCCGAGCAGAAGAAGAA

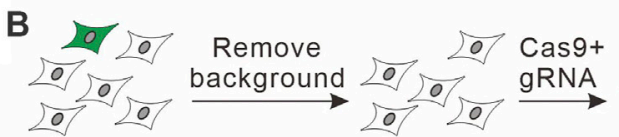

Reporter

cells

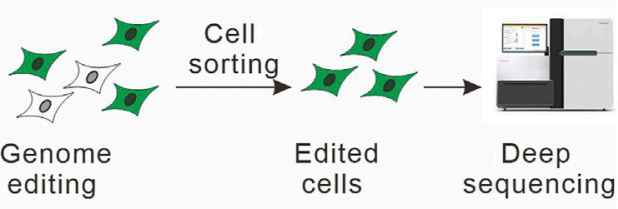

OT31

OT36

OT52
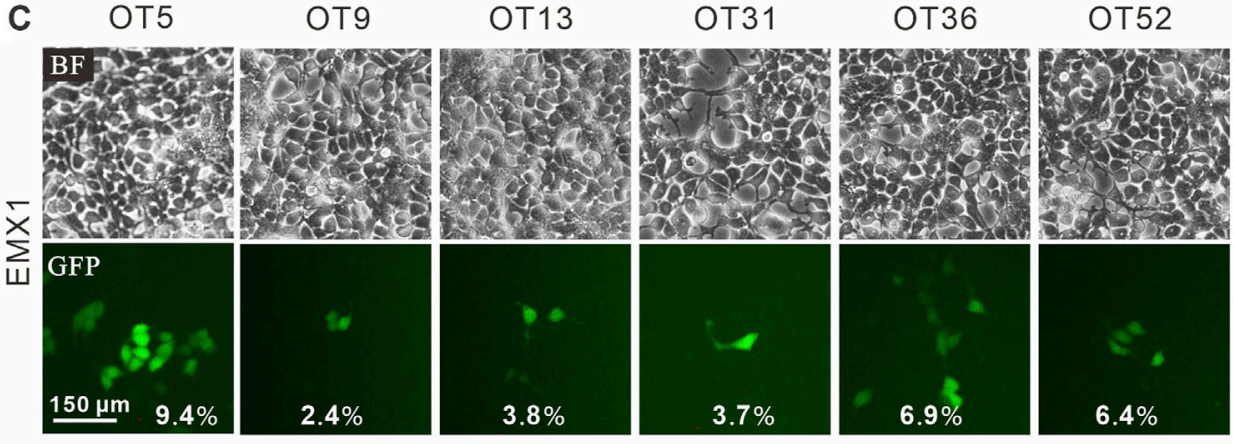

D

OT7

OT19

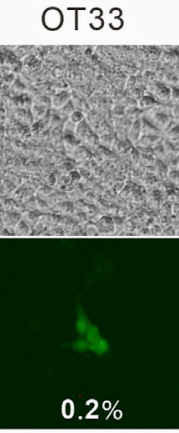

OT49

OT 60
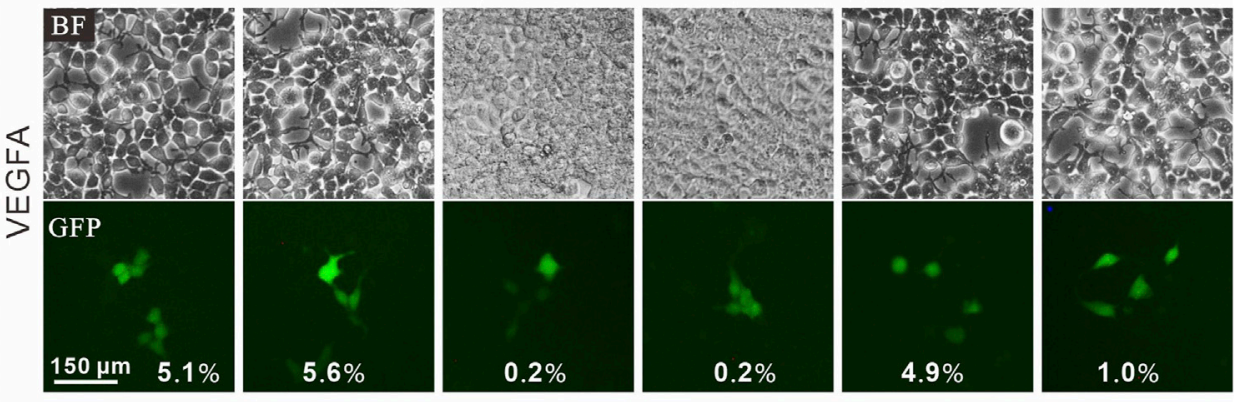

E

On target EMX1-OT5

Indel

GAGTCCGAGCAGAAGAAGAAGGG

ATGAAGTCCGAGGAGAGGAAGAA $\overline{A G G} G T G A G C$

ATGAAGTCCGAGGAGAGGA-- $-------G \mathrm{C}$

ATGAAGTCCGAG----------------AGC

ATGAAGTCCGAGGAGAGG------- GTGAGC

ATG----- --------- GAAAGGGTGAGC

ATGAAGTCCGAGGA------- A $\overline{A G G} G T G A G C$

ATGAAGTCCGAGGAG---- GAA $\overline{A G G} G$ TGAGC

ATGAAGTCCGAGGAGAGGAA--A $\overline{A G G} G T G A G C$

ATGAAGTCCGAGGAGAGGAAAGA $\overline{A A G} G$ GTGAGC

ATGAAGTCCGAGGAGAGGTCACCG GAA $\underline{A G G T G}$

FIGURE 1 | A GFP activation assay for DNA cleavage detection. (A) Schematic diagram of the GFP activation assay. A lentiviral vector contains a CMV-driven GFP. A protospacer followed by a PAM is inserted between ATG and GFP coding sequence, disrupting GFP expression. An example of off-target sequence as well as ontarget gRNA is shown below. (B) The vector is stably integrated into HEK293T cells. Background GFP-positive cells are removed by cell sorting. After genome editing, a portion of cells will restore GFP expression. GFP-positive cells are sorted out and PAM sequences were PCR-amplified for deep-sequencing analysis. (C) Six offtarget sites (for a gRNA targeting EMX1) identified by CIRCLE-seq are selected for validation in cells by the GFP activation assay. (D) Six off-target sites (for a gRNA targeting VEGFA site 1) identified by CIRCLE-seq are selected for validation in cells by the GFP activation assay. (E) Indel sequences are detected by deep sequencing for EMX1-OT5. On target sequence is shown above. Mismatches in the off-target sequence (EMX1-OT5) are shown in blue. The insertions are shown in red. The GFP sequence is shown in green. PAM is underlined. 
TABLE 1 | Off-target sites of EMX1 and VEGFA site 1 in this study.

\begin{tabular}{llr}
\hline Sites & \multicolumn{1}{c}{ Sequence $^{\text {a }}$} & Locus \\
\hline EMX1-On & GAGTCCGAGCAGAAGAAGAAGGG & chr2:72933853-72933875 \\
EMX1-OT5 & AAGTCCGAGGAGAGAAGAAGG & chr1:23384119-23394141 \\
EMX1-OT9 & GAGTACAAGCAGATGAAAACGG & chr10:126391610-126391632 \\
EMX1-OT13 & GAGGCCAAGCAGAAAGAAAAGG & chr7:31861458-31861480 \\
EMX1-OT31 & GACTCCGAGCAGCAGAAGGATGG & chr2:65555376-65555398 \\
EMX1-OT36 & GAGTAGAGCAGAGGAAGAGAGG & chr6:99251280-99251302 \\
EMX1-OT52 & GTGTCAGAGCAGAAAAGAGTGG & chr4:25059119-25059141 \\
VEGFA-On & GGGTGGGGGAGTTGCTCCTGG & chr6:43769554-43769576 \\
VEGFA-OT5 & GGGGGCAGGGAGATTGCTCCTGG & chr18:366708-366730 \\
VEGFA-OT7 & AAGTAAGGGAATTGCTCCTGG & chr16:869350-869372 \\
VEGFA-OT19 & GGGAGGAGAGAGTTGCTCTCTG & chr8:139702078-139702100 \\
VEGFA-OT33 & AGAGGGGTGGATTGTCCAGG & chr20:38342497-38342519 \\
VEGFA-OT49 & GGGGAGGGGGAGATGGCTCCCGG & chr10:113530631-113530653 \\
VEGFA-OT60 & GAGGTGGGTGATTGTCCAGG & chr11:57067754-57067776
\end{tabular}

${ }^{a} P A M$ sequences were shown in green and mismatched nucleotides were shown in red.

locations (Fu et al., 2013; Hsu et al., 2013). Subsequently, several genome-wide unbiased approaches have been developed, including cell-based approaches (such as GUIDE-seq and HTGTS) (Crosetto et al., 2013; Frock et al., 2015; Tsai et al., 2015; Wang et al., 2015) and cell-free approaches (such as CIRCLE-seq) (Kim et al., 2015; Tsai et al., 2017). CIRCLE-seq is supposed to be the most sensitive approach, enabling the identification of rare off-target cleavage events in vitro (Tsai et al., 2017). However, it is a challenge to test whether the rare off-target cleavage events occur in cells. Targeted amplicon sequencing is routinely used to validate off-target mutations, but the error rate of next-generation sequencing places a floor for indel mutation detection of $\sim 0.1 \%$ (Tsai et al., 2017). Therefore, easy and sensitive approaches for DNA cleavage detection in cells are missing.

Previously, we developed a GFP activation assay for CRISPR PAM screening (Hu et al., 2020). In this approach, a target sequence is inserted between ATG and GFP coding sequence, disrupting GFP expression. If Cas9 nucleases cleave the target sequence and induce in-frame mutations, GFP expression will be restored. In this study, we test whether this approach can be used to detect off-target cleavage. We first demonstrate that this approach enables us to verify rare off-target cleavage events that could not be detected in cells before. Next, we demonstrate that this approach enables the detection of multiple alternative SpCas9 PAMs associated with very low cleavage activity. Therefore, this GFP activation assay is a sensitive platform for DNA cleavage detection in cells.

\section{RESULTS}

\section{The GFP Activation Assay for Validation of Off-Targets}

To establish a highly sensitive approach for rare off-target cleavage detection in cells, a previous GFP activation assay was used (Hu et al., 2020). A target sequence (protospacer) with a PAM is inserted between ATG start codon and GFP coding sequence, disrupting GFP expression by frameshift mutation. When Cas9 nucleases cleave the protospacer and generate small insertions/ deletions (indels), a portion of cells will restore the GFP reading frame, leading to GFP expression (Figure 1A). The reporter system was delivered into HEK293T cells by lentiviral infection to generate a stable cell line. Background GFP-positive cells caused by plasmid mutations were removed by cell sorting (Figure 1B).

CIRCLE-seq is the most sensitive approach for genome-wide off-target detection to date (Tsai et al., 2017). Tsai et al. identified up to hundreds of off-target sites for a given target by CIRCLEseq, but they failed to validate a list of off-target sites in cells by using targeted amplicon sequencing (Tsai et al., 2017). It possible that off-target cleavage occurred at these sites but the detection assay was not sensitive enough. To test the power of the GFP activation assay, we first chose two off-target sites (EMX1-OT5 and VEGFA-OT7; Table 1) that can be detected by both CIRCLE-seq and GUIDE-seq (Tsai et al., 2017). GFP-positive cells were observed 2 days after the transfection of SpCas9 with on-target gRNA but not SpCas9 alone (Figures 1C,D; Supplementary Figures S1a,S1b). The GFP-positive cells were sorted out, and the protospacer sequences were PCR-amplified for deep sequencing. Deep sequencing analysis revealed that indels occurred (Figure 1E). These data demonstrated that the GFP activation assay enabled off-target cleavage detection in cells.

We chose 10 additional off-target sites (five for EMX1 and five for VEGFA site 1; Table 1) that were detected by CIRCLE-seq but failed to be validated by targeted amplicon sequencing (Tsai et al., 2017). GFP-positive cells were observed for all of the tested sites 2 days after the transfection of SpCas9 with on-target gRNA but not SpCas9 alone (Figures 1C,D; Supplementary Figures S1a,S1b), indicating that off-target mutations could occur with these sites in cells. Indels were confirmed by deep sequencing (Supplementary Figures S2, S3). These data demonstrated that the GFP activation assay was a highly sensitive platform for DNA cleavage detection.

\section{The GFP Activation Assay for Detection of New Non-Canonical PAMs of SpCas9}

In addition to NGG PAM, SpCas9 also recognizes noncanonical PAMs, including NAG, NCG, NGA, and NNGG (Jiang et al., 2013; Doench et al., 2016). We investigate whether the GFP activation assay is sensitive enough to identify additional PAMs. For this purpose, a protospacer containing $7 \mathrm{nt}$ downstream randomized DNA sequences was inserted between ATG start 


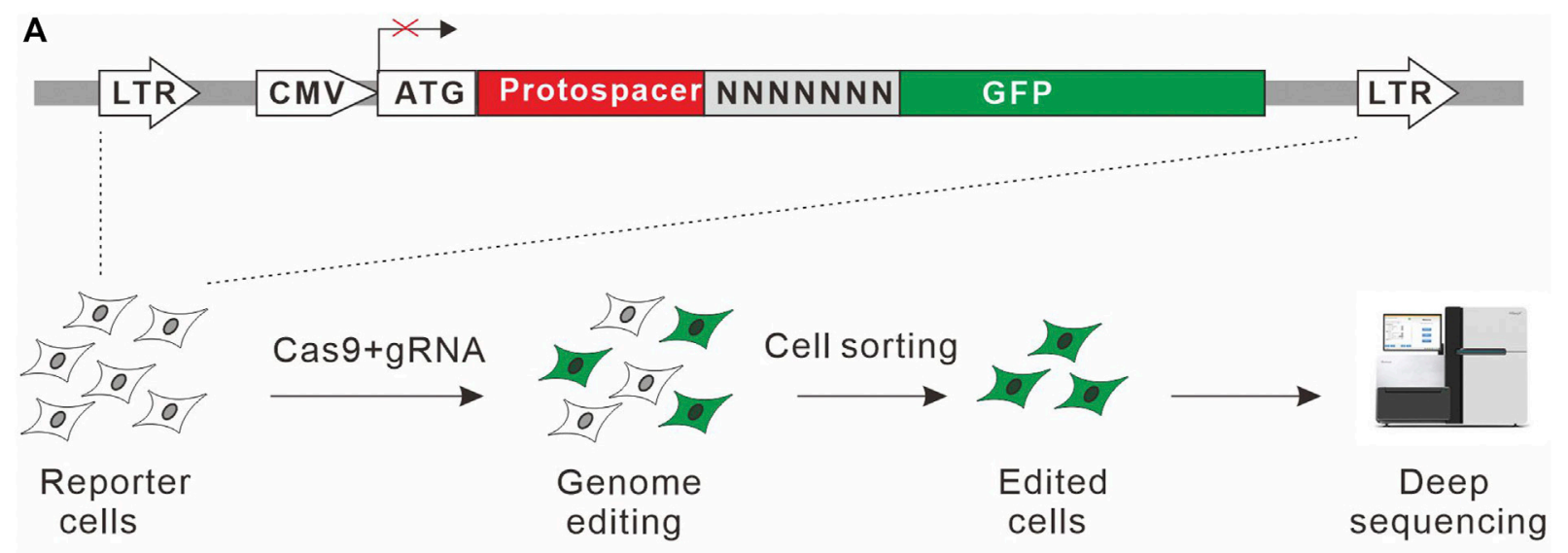

B

SpCas9 only
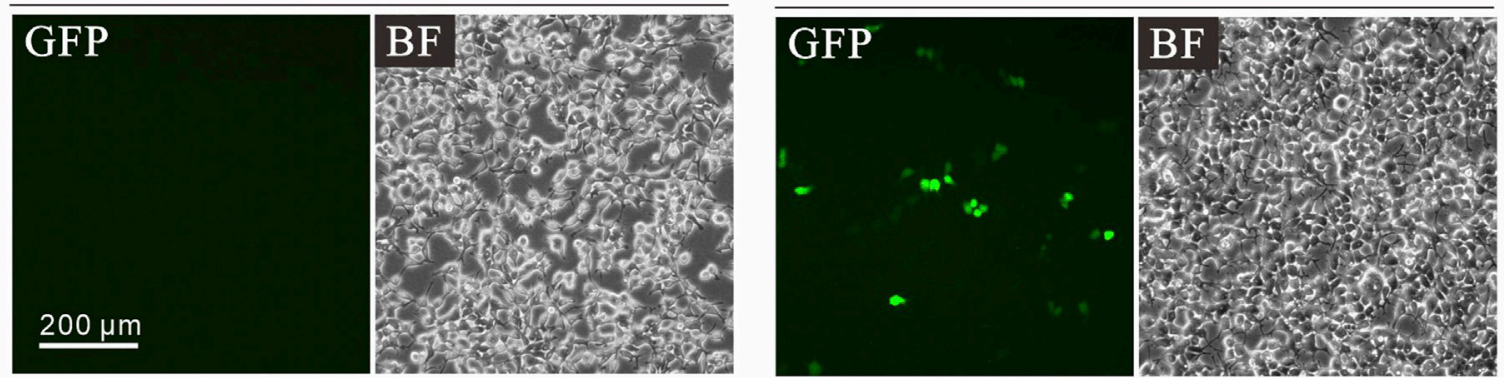

C
ATGGAACGGCTCGGAGATCATCATTGCGNNNNNNNGTGAGCAAG
ATGGAACGGCTCGGAGATCATC. . TGCGTGGAAAGGTGAGCAAG
ATGGAACGGCTCGGAGATCATCA. . GCGGGGCCGAGTGAGCAAG
ATGGAACGGCTCGGAGATC.... TGCGCGGGAGTGTGAGCAAG
ATGGAACGGCTCGGAGATCACCC. . GCGTGGGCGGGTGAGCAAG
ATGGAACGGCTCGGAGATCATCA. . GCGGAGATTAGTGAGCAAG
ATGGAACGGCTCGGAGATC.... . TGCGGTGCTTTGTGAGCAAG
ATGGAACGGCTCGGAGATCATC. . TGCGTAGAGCCGTGAGCAAG
ATGGAACGGCTCGGAGATCATC. . GGCGGGATAGGGTGAGCAAG
ATGGAACGGCTCGGAGATCATCC. . GCGGCGGCGGGTGAGCAAG
ATGGAACGGCTCGGAGATCA. .... GCGGTGGTGGGTGAGCAAG

FIGURE 2|A GFP activation assay for PAM screening. (A) Schematic diagram of the GFP activation assay. A lentiviral vector contains a CMV-driven GFP. A target sequence followed by a 7-bp random sequence is inserted between ATG and GFP coding sequence, disrupting GFP expression. The library is stably integrated into HEK293T cells. After genome editing, a portion of cells will restore GFP expression. GFP-positive cells are sorted out and PAM sequences were PCR-amplified for deepsequencing analysis. (B) Transfection of SpCas9 and gRNA results in GFP expression, while transfection of SpCas9 alone cannot induce GFP expression. (C) Deep-sequencing reveals that targets with multiple PAMs can be edited. GFP sequence is shown in green; insertion mutations are shown in red; 7-bp random sequences are a highlight in yellow. 

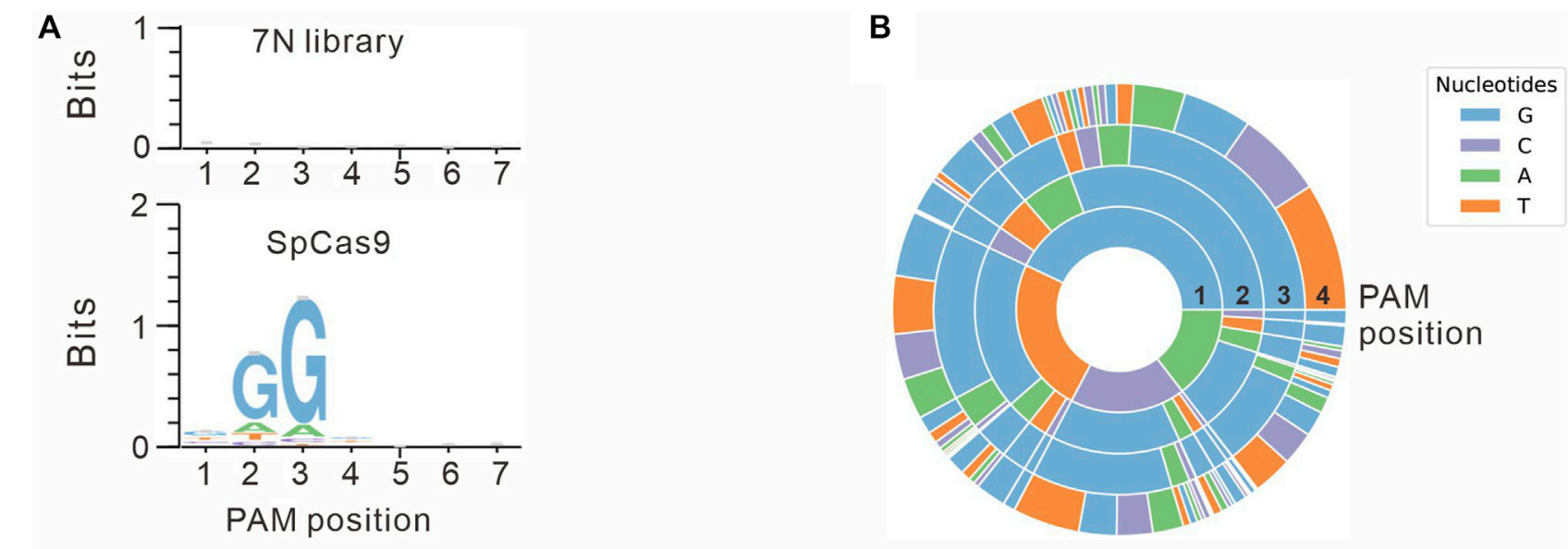

C

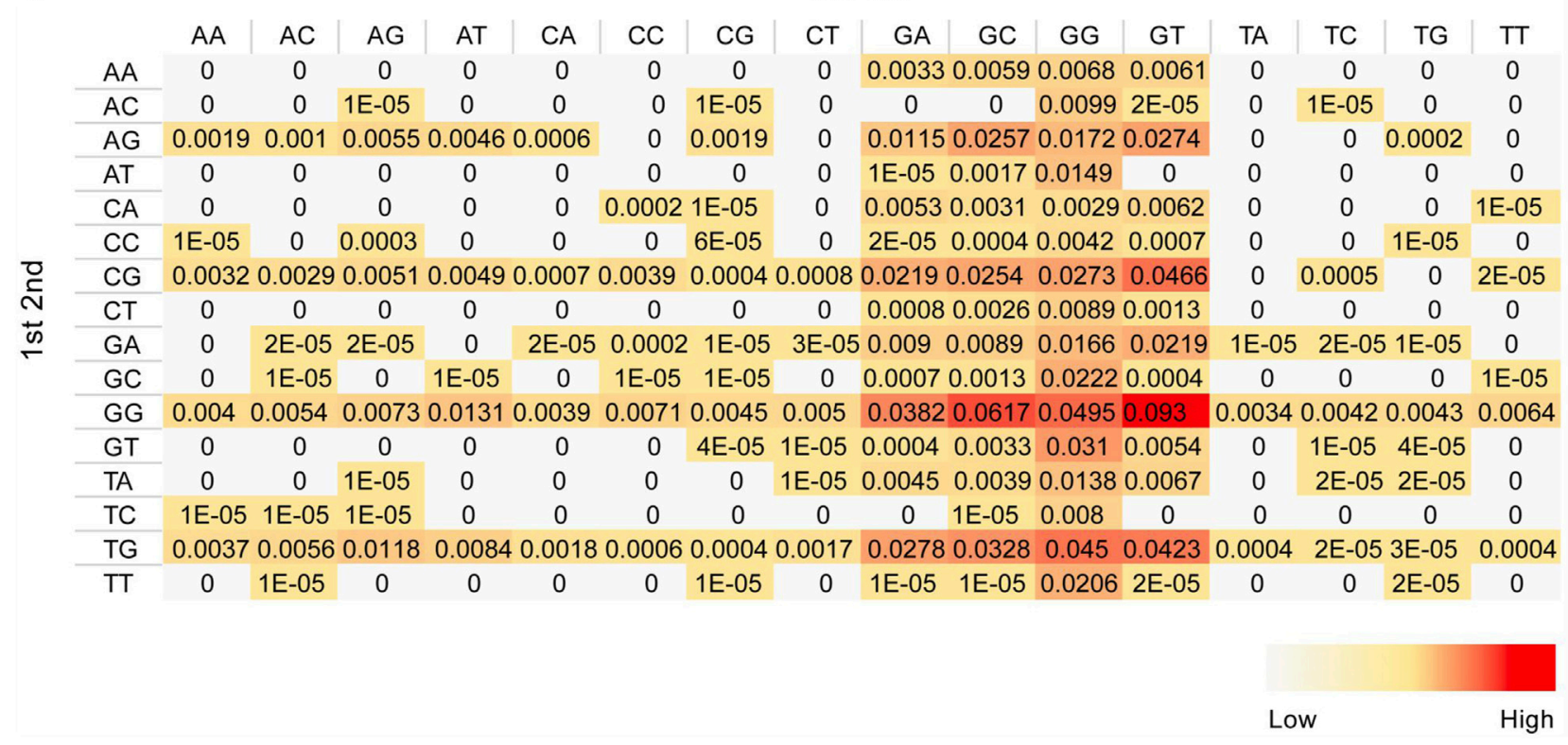

FIGURE 3 |PAM sequence analysis. (A) WebLogo is generated based on the PAM screening assay. The upper panel is the control cells without genome editing. the lower panel is the cells after genome editing. (B) PAM wheel is generated based on the PAM screening assay. (C) Heat map for efficiency ratio of top all 256 PAMs. The 1-2 PAM sequences are shown on the left, and the 3-4 PAM sequences are shown on the top.

codon and GFP coding sequence, disrupting GFP expression (Figure 2A). The GFP reporter construct was stably inserted into the genome. Quantitative PCR (qPCR) revealed that there were average 3.82 copies of construct per cells. GFP-positive cells could be observed 2 days after the transfection of SpCas9 with the corresponding gRNA but not SpCas9 alone (Figure 2B). The GFP-positive cells were sorted out, and the protospacer with randomized DNA sequences were PCR-amplified for deep sequencing. Deep sequencing analysis revealed that indels associated with different PAMs could be detected (Figure 2C).

Next, we systematically analyzed the PAM sequences recognized by SpCas9 from deep sequencing data. Only inframe mutations were considered as novel mutations induced by SpCas9, thus minimizing the background mutations derived from library construction or deep sequencing. Since indels could disrupt the randomized DNA sequences, GCG triple-nucleotide was used to fix the $7 \mathrm{nt}$ randomized DNA sequences (Figure 2C). Both WebLogo and PAM wheel captured the canonical NGG as the most enriched PAM, but the sequences other than NGG were also observed (Figures 3A,B). The nucleotide preference at position 1-4 was not random. We analyzed the PAM frequencies for all possible NNNN PAM sequences (Figure 3C). The top 74 PAMs (frequency over $0.1 \%$ ) included all NNGG, NAGN, NGAN, and GGYN PAMs (Supplementary Figure S4). The most efficient PAM was NGGN, as expected, followed by NNGG, NAGN, NGAN, and GGYN (Figure 3C). Interestingly, 


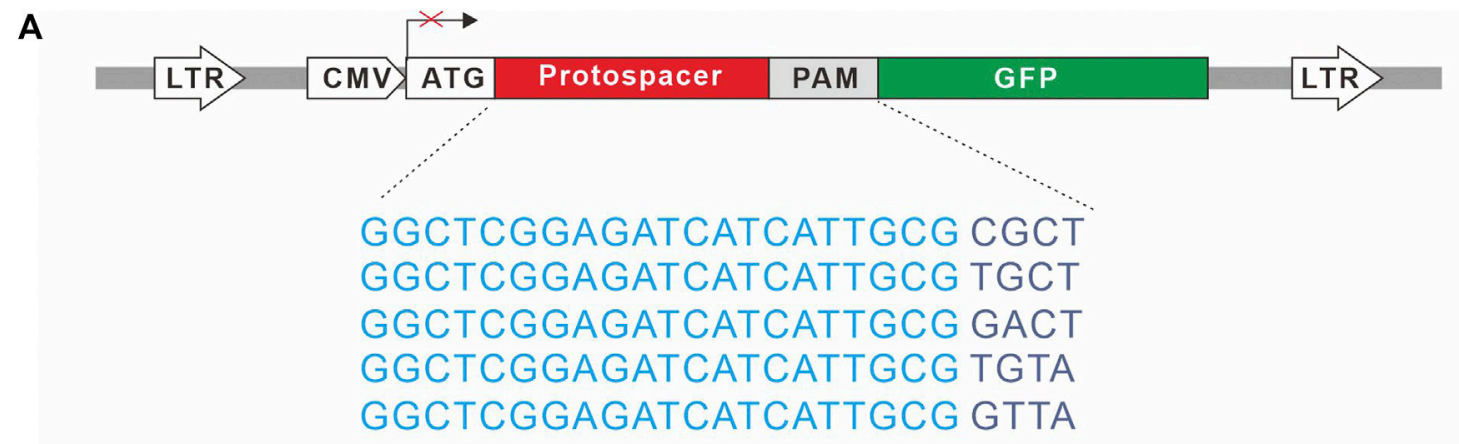

B
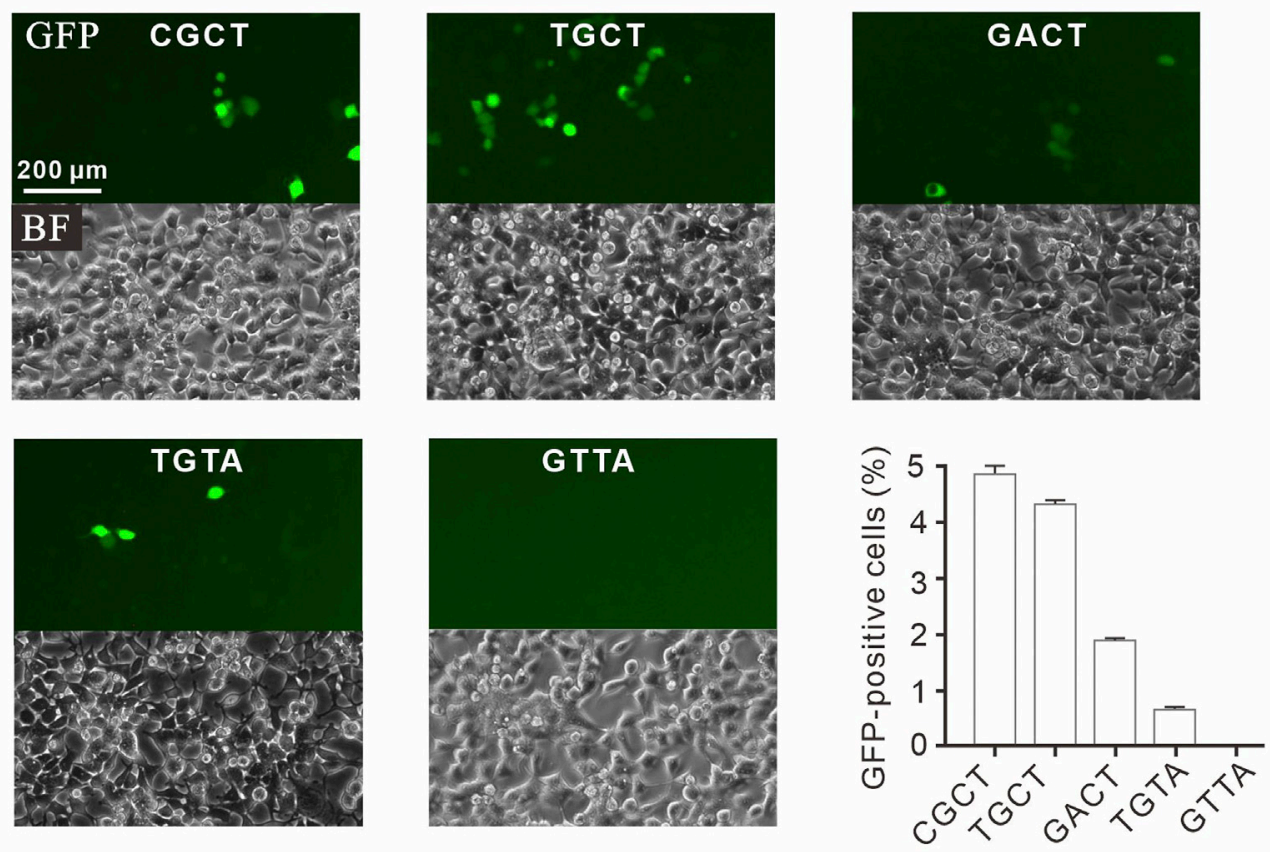

C
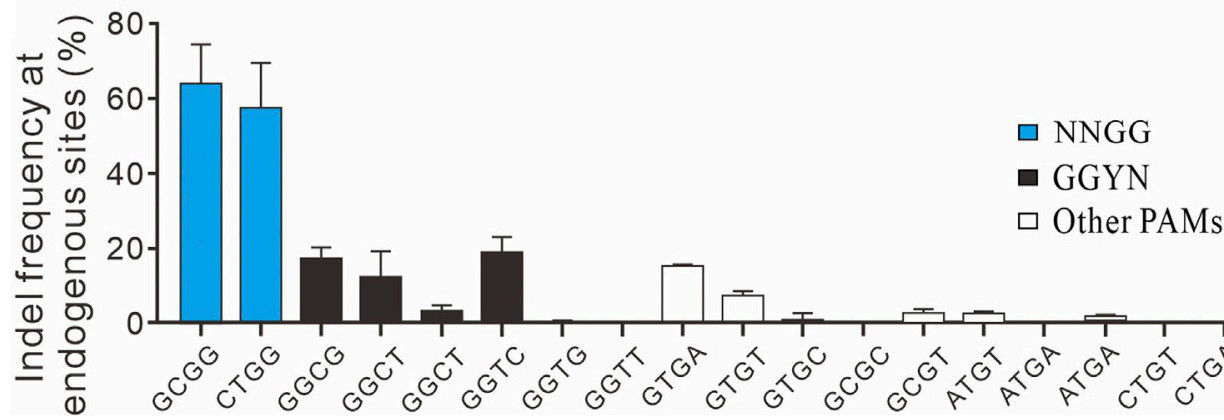

FIGURE 4 IValidation of noncanonical PAMs. (A) Schematic diagram of the GFP activation assay with five targets shown below. (B) Five GFP reporter constructs with noncanonical PAMs are isolated from the PAM library and tested for genome editing. Quantification is shown on the bottom right. $n=3$. (C) Indel frequency of endogenous target sequences associated with noncanonical PAMs. $n=3$. 
some noncanonical PAMs such as GTGG, GCGG, and GAGT displayed comparable efficiency to NGGN PAMs.

To confirm novel PAMs identified here, we isolated four GFP reporter constructs with different PAM sequences (CGCT, TGCT, GACT, and TGTA) from the PAM library and established stable cell lines for each construct (Figure 4A). Transfection of SpCas9 with gRNA induced GFP expression for all of them, indicating that cleavage occurred with these PAMs (Figure 4B). We also isolated a construct with GTTA PAM as a negative control. Consistent with the PAM screening results, GFP-positive cells could not be observed with GTTA PAM (Figure 4B). We further tested 21 endogenous targets with noncanonical PAMs. Two NNGG PAMs displayed very high activity, with indel rates of 64.3 and $57.6 \%$ for GCGG and CTGG, respectively (Figure 4C). Three GGYN PAMs (GGCG, GGCT, and GGTC) as well as GTGA and GTGT PAMs also displayed significant activity. Other PAMs only displayed minimal activity. These novel PAMs are useful for in silico prediction of potential off-target sites.

\section{DISCUSSION}

In this study, we demonstrate that the GFP activation assay is a highly sensitive platform for DNA cleavage detection in cells. Targeted amplicon sequencing is routinely used to detect DNA cleavage, but the error rate of next-generation sequencing places a floor for indel mutation detection of $\sim 0.1 \%$ (Tsai et al., 2017). Our GFP activation assay requires a cell sorting step to enrich the cleavage events before targeted amplicon sequencing, which increases the sensitivity. GFP-disruption assay can be used for detection of DNA cleavage (Permyakova et al., 2019), but it cannot detect the rare cleavage events due to the background of GFPnegative cells. In addition, GFP-disruption assay does not allow us to test non-GFP DNA sequence. "Traffic light" system is an elegant design for detection of DNA cleavage in cells (Certo et al., 2011). This system contains a GFP cassette disrupted by a target sequence and an RFP cassette which is out of frame. If DNA cleavage occurs and DSB is repaired by NHEJ, the RFP expression will be activated. The sensitivity of this system should be comparable to our assay.

In the GFP activation assay, the GFP reporter construct is inserted into the genome by lentiviruses, which preferentially integrate into transcriptionally active regions (Schröder et al., 2002; Mitchell et al., 2004; Kim et al., 2017). These regions are much more accessible for the CRISPR/Cas9 machinery. One limitation of this assay is that it may not reflect the real situation in the therapeutically relevant cells, where endogenous off-target sites may be not accessible for Cas9 nucleases. On the other hand, GFP activation assay has an advantage over detection at endogenous loci. When Cas9+gRNA are delivered into the human body by adeno-associated virus (AAV) (Wang et al., 2019b), AAV can also infect therapeutically irrelevant cell types, where the off-target sites may be open. It is not safe enough to only consider therapeutically relevant cells.

The GFP activation assay requires to clone every potential off-target site into the vector and establishes stable cell lines, which is timeconsuming. For clinical use with CRISPR/Cas9, it deserves to test very rare cleavage events happened at off-targets. It is also possible to synthesize all potential off-targets with microarray and clone them into the vector to establish a library. The library is stably integrated into chromosome. Transfection of Cas9+gRNA can induce GFP expression. GFP-positive cells are sorted out and synthesized off-targets are PCRamplified for deep sequencing. This procedure may allow highthroughput test of potential off-targets with GFP activation assay.

SpCas9 is the most extensively studied and applied system to date due to its high efficiency and simple PAM requirement (Cong et al., 2013; Mali et al., 2013). In addition to NGG PAM, several noncanonical PAMs including NAG, NCG, NGA, and NNGG have been identified (Jiang et al., 2013; Doench et al., 2016). These noncanonical PAMs are associated with low cleavage activity, but targets associated with them must be considered as potential off-target sites. Therefore, it is crucial to identify additional noncanonical PAMs for SpCas9. The GFP activation assay enables to identify multiple noncanonical PAMs, dramatically extending the list of noncanonical SpCas9 PAMs. We anticipate that the GFP activation assay is also useful for other applications that require highly sensitive DNA cleavage detection, such as the test of new genome tools.

\section{METHODS}

\section{Cell Culture and Transfection}

HEK293T cells were maintained in Dulbecco's Modified Eagle Medium (DMEM) supplemented with 10\% FBS (Gibco) and 1\% antibiotics at $37^{\circ} \mathrm{C}$ with $5 \% \mathrm{CO}_{2}$. For the PAM library screen, HEK293T cells were plated into $10 \mathrm{~cm}$ dishes, and transfected at $\sim 60 \%$ confluency with Cas9-gRNA-expressing plasmid (15 $\mu \mathrm{g}$ ) using Lipofectamine 2000 (Life Technologies). For PAM validation at endogenous sites, HEK293T cells were seeded on 48-well plates and transfected with Cas9-gRNA-expressing plasmid (500 ng) using Lipofectamine 2000 (Life Technologies).

\section{The PAM Library Construction}

The DNA oligonucleotides containing a target sequence followed by random sequences and flanking homologous sequences (for Gibson Assembly) were synthesized from GENEWIZ (Suzhou, China). Full-length oligonucleotides were PCR-amplified using Q5 High-Fidelity 2X Master Mix (NEB), size-selected using a 3\% agarose gel EX (Life Technologies, Qiagen), and purified using MinElute Gel Extraction Kit (Qiagen). PCR products were cloned into a lentiviral vector by Gibson Assembly (NEB) and purified with Agencourt AMPure XP SPRI beads (Beckman Coulter). The Gibson Assembly products were electroporated into MegaX $\mathrm{DH}_{10 \mathrm{~B}}{ }^{\mathrm{TM}} \mathrm{T} 1^{\mathrm{R}}$ Electrocomp ${ }^{\mathrm{TM}}$ Cells (Invitrogen) using a GenePulser (BioRad). The bacteria were added into recovery media and grew at $32^{\circ} \mathrm{C}, 225 \mathrm{rpm}$ for $14 \mathrm{~h}$. The plasmid DNA was extracted from bacteria using Endotoxin-Free Plasmid Maxiprep (Qiagen). The plasmid sequence is shown in Supplementary Figure S5. All primers and gRNA sequences used in this study were listed in Supplementary Table S1.

\section{Lentivirus Production}

For PAM library packaging, HEK293T cells were seeded in three $10 \mathrm{~cm}$ dishes and transfected at $\sim 40 \%$ confluency. For each dish, $12 \mu \mathrm{g}$ of PAM library plasmid, $9 \mu \mathrm{g}$ of psPAX2, and $3 \mu \mathrm{g}$ of pMD2.G were transfected with $60 \mu \mathrm{L}$ of Lipofectamine 2000 (Life Technologies). Viruses were harvested twice at 48 and $72 \mathrm{~h}$ post-transfection. The 
viruses were concentrated using PEG8000 (no. LV810A-1, SBI, Palo Alto, CA), dissolved in PBS and stored at $-80^{\circ} \mathrm{C}$. For single PAM reporter construct packaging, HEK293T cells were seeded into 6-well plates, $1.2 \mu \mathrm{g}$ of PAM reporter plasmid, $0.9 \mu \mathrm{g}$ of psPAX2, and $0.3 \mu \mathrm{g}$ of pMD2.G were transfected with $5 \mu \mathrm{L}$ of Lipofectamine 2000. Viruses were harvested twice at 48 and $72 \mathrm{~h}$ post-transfection.

\section{PAM Library Screening Assay}

HEK293T cells were plated into a $15 \mathrm{~cm}$ dish at $~ 30 \%$ confluence. After $24 \mathrm{~h}$, cells were infected with PAM library lentiviruses with at least 1000-fold coverage of each PAM. $24 \mathrm{~h}$ after infection, the cells were selected with $2 \mu \mathrm{g} / \mathrm{ml}$ of puromycin for 5 days. To remove plasmid mutations that induce GFP expression, the GFP negative cells were sorted out with a MoFlo XDP machine (Beckman Coulter) and seeded into $10 \mathrm{~cm}$ dishes. The residual GFP-positive cells were removed pipette tips under microscope. For the PAM screen, PAM library cells were transfected with Cas9-gRNA expressing plasmid for 3 days. The GFP-positive cells were sorted out by MoFlo XDP machine and the genomic DNA was isolated using TIANamp Genomic DNA Kit (TIANGEN) following the manufacturer's instructions. DNA target sites were PCR-amplified by nested PCR with Q5 High-Fidelity 2X Master Mix (NEB). First, the target region was PCR-amplified using primers Deep-F1/R1 with 25 cycles; second, $3 \mu \mathrm{L}$ of PCR products from the first step were used as a template and amplified by primers P5-adapter-F and P7-adapter-R for 15 cycles. The PCR products were purified using the Gel Extraction Kit (Qiagen) and sequenced on Illumina HiSeq $\mathrm{X}$ by 150 -bp paired-end sequencing.

\section{PAM Sequence Analysis}

Twenty base-pair sequences (AAGCCTTGTTTGCCACCATG/ GTGAGCAAGGGCGAGGAGCT) flanking the target sequence (GAACGGCTCGGAGATCATCATTGCGNNNNNNN) were used to fix the target sequence. Only target sequences with inframe mutations were used for PAM analysis. GCG and GTG AGCAAGGGCGAGGAGCT sequences were used to fix a 7-bp random sequence. Only intact 7-bp random sequences were used for PAM analysis. The 7-bp random sequences were extracted and visualized by WebLog3 (Crooks et al., 2004) and wheel chart (Leenay et al., 2016) to demonstrate PAMs.

\section{Plasmid Constructs and DNA Cleavage Detection}

Plasmids containing specific PAM sequences were isolated from the PAM library. Plasmids of EMX1-OT and VEGFA-OT construction: vector backbone of the plasmid containing specific PAM sequences was PCR-amplified using primers EMX1/ VEGFA-OT-F/cozak-R, followed by phosphorylation with T4 Polynucleotide Kinase (NEB) and religation with T4 DNA ligase (NEB). Each plasmid was packed into lentiviruses to generate a stable cell line. To remove plasmid mutations that induce GFP expression, the GFP-negative cells were sorted out by the MoFlo XDP machine (Beckman Coulter). The cells were seeded into 24-well and transfected with 800 ng of SpCas9-gRNAexpressing plasmid (PX459, addgene \#118632) by Lipofectamine
2000 (Life Technologies). Five days after transfection, the GFPpositive cells were measured on the Calibur instrument (BD). Data were analyzed using FlowJo. Besides, GFP-positive cells of EMX1OT, and VEGFA-OT were sorted out by the MoFlo XDP machine (Beckman Coulter). The genomic DNA was isolated and target sites were amplified by nested PCR and extracted by Gel Extraction Kit (QIAGEN). The amplicons are prepared for deep sequencing.

\section{Test of PAM Activity at Endogenous Sites}

HEK293T cells were seeded on 24-well plates and transfected with epiCRISPR(4) constructs expressing SpCas9 and gRNA followed by puromycin selection $(2 \mu \mathrm{g} / \mathrm{ml})$ for 6 days. The genomic DNA was isolated, and the target sites were PCRamplified by nested PCR for deep sequencing.

\section{Quantification of GFP Reporter Copy Number}

The genomic DNA was isolated from GFP activation cells using TIANamp Genomic DNA Kit (TIANGEN) following the manufacturer's instructions. To generate standard curve, GFP reporter plasmid was serially diluted to final concentrations of $10^{9}, 10^{8}, 10^{7}, 10^{6}, 10^{5}, 10^{4}, 10^{3}, 10^{2}$ copies/ $\mu \mathrm{L}$. Quantitative PCR were performed with CFX Connect Real-Time System (Bio-Rad) and 2X SYBR Green qPCR Master Mix (APExBIO). Use the following parameters: 1 cycle at $95^{\circ} \mathrm{C}$ for $2 \mathrm{~min}$, followed by 40 cycles of $95^{\circ} \mathrm{C}$ for $15 \mathrm{~s}, 55^{\circ} \mathrm{C}$ for $30 \mathrm{~s}$, and $72^{\circ} \mathrm{C}$ for $30 \mathrm{~s}$. Each sample was quantified in triplicate. The same parameters were used to quantify GFP copy numbers in the genomic DNA. Absolute transgene copy numbers were calculated with $\mathrm{C}_{\mathrm{q}}$ values and dilution factor based on standard curve.

\section{Quantification and Statistical Analysis}

All the data are shown as the mean \pm S.D. Statistical analyses were conducted using Microsoft Excel.

\section{DATA AVAILABILITY STATEMENT}

The data presented in this study are deposited in the NCBI SRA BioProject repository, accession number is PRJNA773240.

\section{AUTHOR CONTRIBUTIONS}

ZH, DW, and SG: performed the experiments; CZ: analyzed the data; SO: edited the writing; YW: designed the experiments and wrote the article; WZ: supervised the project and offered grant support. All authors read and approved the final article.

\section{FUNDING}

This work was supported by grants from the National Natural Science Foundation of China (82070258, 81870199), the Foundation for Innovative Research Group of the National Natural Science Foundation of China (31521003), Science and 
Technology Research Program of Shanghai (19DZ2282100), Open Research Fund of State Key Laboratory of Genetic Engineering, Fudan University (No. SKLGE-2104), the Scientific Research Foundation of Peking University Shenzhen Hospital KYQD202100X.

\section{REFERENCES}

Certo, M. T., Ryu, B. Y., Annis, J. E., Garibov, M., Jarjour, J., Rawlings, D. J., et al. (2011). Tracking Genome Engineering Outcome at Individual DNA Breakpoints. Nat. Methods 8, 671-676. doi:10.1038/nmeth.1648

Cong, L., Ran, F. A., Cox, D., Lin, S., Barretto, R., Habib, N., et al. (2013). Multiplex Genome Engineering Using CRISPR/Cas Systems. Science 339, 819-823. doi:10.1126/science.1231143

Crooks, G. E., Hon, G., Chandonia, J. M., and Brenner, S. E. (2004). WebLogo: A Sequence Logo Generator. Genome Res. 14, 1188-1190. doi:10.1101/gr.849004

Crosetto, N., Mitra, A., Silva, M. J., Bienko, M., Dojer, N., Wang, Q., et al. (2013). Nucleotide-resolution DNA Double-Strand Break Mapping by NextGeneration Sequencing. Nat. Methods 10, 361-365. doi:10.1038/nmeth.2408

Doench, J. G., Fusi, N., Sullender, M., Hegde, M., Vaimberg, E. W., Donovan, K. F., et al. (2016). Optimized sgRNA Design to Maximize Activity and Minimize Off-Target Effects of CRISPR-Cas9. Nat. Biotechnol. 34, 184-191. doi:10.1038/nbt.3437

Frock, R. L., Hu, J., Meyers, R. M., Ho, Y.-J., Kii, E., and Alt, F. W. (2015). Genomewide Detection of DNA Double-Stranded Breaks Induced by Engineered Nucleases. Nat. Biotechnol. 33, 179-186. doi:10.1038/nbt.3101

Fu, Y., Foden, J. A., Khayter, C., Maeder, M. L., Reyon, D., Joung, J. K., et al. (2013). High-frequency Off-Target Mutagenesis Induced by CRISPR-Cas Nucleases in Human Cells. Nat. Biotechnol. 31, 822-826. doi:10.1038/nbt.2623

Fu, Y., Sander, J. D., Reyon, D., Cascio, V. M., and Joung, J. K. (2014). Improving CRISPR-Cas Nuclease Specificity Using Truncated Guide RNAs. Nat. Biotechnol. 32, 279-284. doi:10.1038/nbt.2808

Hsu, P. D., Scott, D. A., Weinstein, J. A., Ran, F. A., Konermann, S., Agarwala, V., et al. (2013). DNA Targeting Specificity of RNA-Guided Cas9 Nucleases. Nat. Biotechnol. 31, 827-832. doi:10.1038/nbt.2647

Hu, Z., Wang, S., Zhang, C., Gao, N., Li, M., Wang, D., et al. (2020). A Compact Cas9 Ortholog from Staphylococcus Auricularis (SauriCas9) Expands the DNA Targeting Scope. Plos Biol. 18, e3000686. doi:10.1371/journal.pbio.3000686

Hwang, W. Y., Fu, Y., Reyon, D., Maeder, M. L., Tsai, S. Q., Sander, J. D., et al. (2013). Efficient Genome Editing in Zebrafish Using a CRISPR-Cas System. Nat. Biotechnol. 31, 227-229. doi:10.1038/nbt.2501

Jiang, W., Bikard, D., Cox, D., Zhang, F., and Marraffini, L. A. (2013). RNA-guided Editing of Bacterial Genomes Using CRISPR-Cas Systems. Nat. Biotechnol. 31, 233-239. doi:10.1038/nbt.2508

Jinek, M., Chylinski, K., Fonfara, I., Hauer, M., Doudna, J. A., and Charpentier, E. (2012). A Programmable Dual-RNA-Guided DNA Endonuclease in Adaptive Bacterial Immunity. Science 337, 816-821. doi:10.1126/science.1225829

Kim, D., Bae, S., Park, J., Kim, E., Kim, S., Yu, H. R., et al. (2015). Digenome-seq: Genome-wide Profiling of CRISPR-Cas9 Off-Target Effects in Human Cells. Nat. Methods 12 (3), 237-243. 231 p following 243. doi:10.1038/nmeth.3284

Kim, H. K., Song, M., Lee, J., Menon, A. V., Jung, S., Kang, Y.-M., et al. (2017). In Vivo high-throughput Profiling of CRISPR-Cpf1 Activity. Nat. Methods 14, 153-159. doi:10.1038/nmeth.4104

Leenay, R. T., Maksimchuk, K. R., Slotkowski, R. A., Agrawal, R. N., Gomaa, A. A., Briner, A. E., et al. (2016). Identifying and Visualizing Functional PAM Diversity across CRISPR-Cas Systems. Mol. Cel. 62, 137-147. doi:10.1016/j.molcel.2016.02.031

Lin, Y., Cradick, T. J., Brown, M. T., Deshmukh, H., Ranjan, P., Sarode, N., et al. (2014). CRISPR/Cas9 Systems Have Off-Target Activity with Insertions or Deletions between Target DNA and Guide RNA Sequences. Nucleic Acids Res. 42, 7473-7485. doi:10.1093/nar/gku402

Mali, P., Yang, L., Esvelt, K. M., Aach, J., Guell, M., DiCarlo, J. E., et al. (2013). RNA-guided Human Genome Engineering via Cas9. Science 339, 823-826. doi:10.1126/science. 1232033

Mitchell, R. S., Beitzel, B. F., Schroder, A. R., Shinn, P., Chen, H., Berry, C. C., et al. (2004). Retroviral DNA Integration: ASLV, HIV, and MLV Show Distinct Target Site Preferences. Plos Biol. 2, E234. doi:10.1371/journal.pbio.0020234

\section{SUPPLEMENTARY MATERIAL}

The Supplementary Material for this article can be found online at: https://www.frontiersin.org/articles/10.3389/fcell.2021.771248/ full\#supplementary-material

Permyakova, N. V., Sidorchuk, Y. V., Marenkova, T. V., Khozeeva, S. A., Kuznetsov, V. V., Zagorskaya, A. A., et al. (2019). CRISPR/Cas9-mediated Gfp Gene Inactivation in Arabidopsis Suspension Cells. Mol. Biol. Rep. 46, 5735-5743. doi:10.1007/s11033-019-05007-y

Ran, F. A., Hsu, P. D., Lin, C.-Y., Gootenberg, J. S., Konermann, S., Trevino, A. E., et al. (2013a). Double Nicking by RNA-Guided CRISPR Cas9 for Enhanced Genome Editing Specificity. Cell 154, 1380-1389. doi:10.1016/j.cell.2013.08.021

Ran, F. A., Hsu, P. D., Wright, J., Agarwala, V., Scott, D. A., and Zhang, F. (2013b). Genome Engineering Using the CRISPR-Cas9 System. Nat. Protoc. 8, 2281-2308. doi:10.1038/nprot.2013.143

Schröder, A. R. W., Shinn, P., Chen, H., Berry, C., Ecker, J. R., and Bushman, F. (2002). HIV-1 Integration in the Human Genome Favors Active Genes and Local Hotspots. Cell 110, 521-529. doi:10.1016/s0092-8674(02)00864-4

Tsai, S. Q., and Joung, J. K. (2016). Defining and Improving the Genome-wide Specificities of CRISPR-Cas9 Nucleases. Nat. Rev. Genet. 17, 300-312. doi:10.1038/nrg.2016.28

Tsai, S. Q., Nguyen, N. T., Malagon-Lopez, J., Topkar, V. V., Aryee, M. J., and Joung, J. K. (2017). CIRCLE-seq: a Highly Sensitive In Vitro Screen for Genome-wide CRISPRCas9 Nuclease Off-Targets. Nat. Methods 14, 607-614. doi:10.1038/nmeth.4278

Tsai, S. Q., Zheng, Z., Nguyen, N. T., Liebers, M., Topkar, V. V., Thapar, V., et al. (2015). GUIDE-seq Enables Genome-wide Profiling of Off-Target Cleavage by CRISPR-Cas Nucleases. Nat. Biotechnol. 33, 187-197. doi:10.1038/nbt.3117

Wang, B., Wang, Z., Wang, D., Zhang, B., Ong, S.-G., Li, M., et al. (2019a). krCRISPR: an Easy and Efficient Strategy for Generating Conditional Knockout of Essential Genes in Cells. J. Biol. Eng. 13, 35. doi:10.1186/s13036-019-0150-y

Wang, D., Tai, P. W. L., and Gao, G. (2019b). Adeno-associated Virus Vector as a Platform for Gene Therapy Delivery. Nat. Rev. Drug Discov. 18, 358-378. doi:10.1038/s41573-019-0012-9

Wang, X., Wang, Y., Wu, X., Wang, J., Wang, Y., Qiu, Z., et al. (2015). Unbiased Detection of Off-Target Cleavage by CRISPR-Cas9 and TALENs Using IntegraseDefective Lentiviral Vectors. Nat. Biotechnol. 33, 175-178. doi:10.1038/nbt.3127

Wang, Y., Liang, P., Lan, F., Wu, H., Lisowski, L., Gu, M., et al. (2014). Genome Editing of Isogenic Human Induced Pluripotent Stem Cells Recapitulates Long QT Phenotype for Drug Testing. J. Am. Coll. Cardiol. 64, 451-459. doi:10.1016/ j.jacc.2014.04.057

Wang, Y., Zhang, W. Y., Hu, S., Lan, F., Lee, A. S., Huber, B., et al. (2012). Genome Editing of Human Embryonic Stem Cells and Induced Pluripotent Stem Cells with Zinc finger Nucleases for Cellular Imaging. Circ. Res. 111, 1494-1503. doi:10.1161/circresaha.112.274969

Xie, Y., Wang, D., Lan, F., Wei, G., Ni, T., Chai, R., et al. (2017). An Episomal VectorBased CRISPR/Cas9 System for Highly Efficient Gene Knockout in Human Pluripotent Stem Cells. Sci. Rep. 7, 2320. doi:10.1038/s41598-017-02456-y

Conflict of Interest: The authors declare that the research was conducted in the absence of any commercial or financial relationships that could be construed as a potential conflict of interest.

Publisher's Note: All claims expressed in this article are solely those of the authors and do not necessarily represent those of their affiliated organizations, or those of the publisher, the editors and the reviewers. Any product that may be evaluated in this article, or claim that may be made by its manufacturer, is not guaranteed or endorsed by the publisher.

Copyright (c) $2021 \mathrm{Hu}$, Zhang, Wang, Gao, Ong, Wang and Zheng. This is an openaccess article distributed under the terms of the Creative Commons Attribution License (CC BY). The use, distribution or reproduction in other forums is permitted, provided the original author(s) and the copyright owner(s) are credited and that the original publication in this journal is cited, in accordance with accepted academic practice. No use, distribution or reproduction is permitted which does not comply with these terms. 